\title{
Le papillon
}

- Citation : CMAJ 2021 January 4;193:E27-8. doi : 10.1503/cmaj.202094-f

Voir la version anglaise de l'article ici : www.cmaj.ca/lookup/doi/10.1503/cmaj.202094

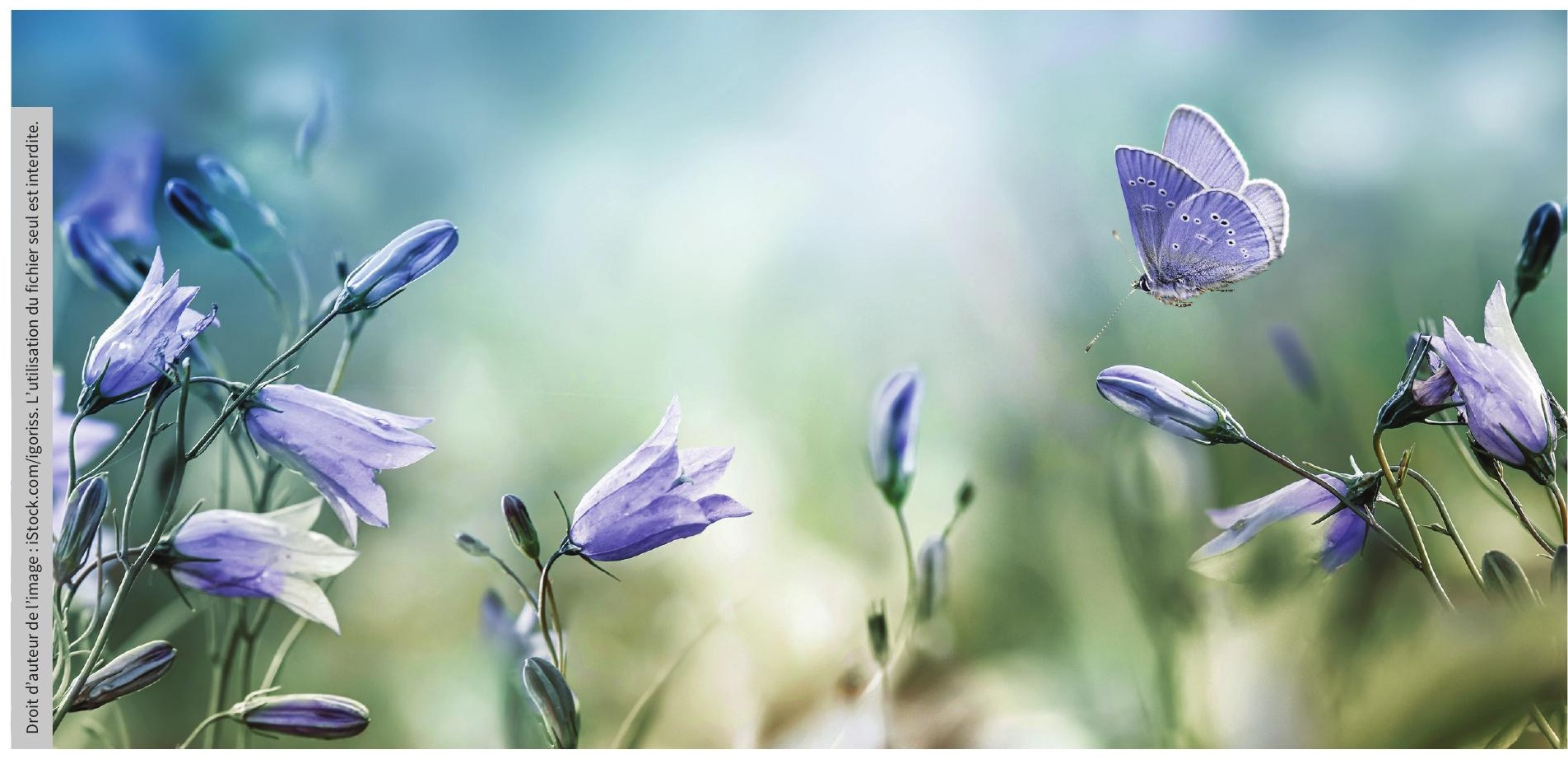

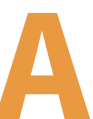
près son diagnostic, je n'ai cessé de me poser des questions. La mammographie tardive me tourmentait. Je me sentais tellement coupable. Je passais parfois la moitié de la nuit éveillée : j'imaginais sa peur, je pensais à sa petite fille, à sa mère aux yeux rougis...

Elle était dans la vingtaine. Aucun antécédent familial, aucun facteur de risque. Le jour où elle m'a dit ressentir une lourdeur à la poitrine, je n'ai palpé aucune bosse. Jeune et active, un enfant dans les bras, elle se demandait si c'était une élongation musculaire.

Ce n'est que quelques semaines plus tard lors d'un second examen qu'à mon grand désarroi, j'ai palpé une masse. Déstabilisée, étourdie et rongée par la culpabilité, je lui ai prescrit une mammographie d'urgence. Comment avais-je pu passer à côté?
«Nous avons détecté une tumeur maligne volumineuse, m'a dit d'un ton grave le radiologiste au téléphone. Je lui ai demandé de se rendre à ton bureau sans attendre. " Sous le choc, j'ai réalisé que je n'avais qu'environ trois minutes pour me préparer à lui annoncer la terrible nouvelle, et ce, au beau milieu d'une matinée chargée. Comment annoncer ce diagnostic à une jeune femme seule, sans son mari pour l'épauler? Comment lui expliquer sans avoir discuté avec le département d'oncologie au préalable, sans avoir prévu plus de temps pour elle à la fin de la journée? Elle s'est présentée avec sa sœur. Les deux étaient déjà très inquiètes. J'ai eu du mal à rester calme et à garder la tête froide. Je ne me souviens plus exactement comment je lui ai annoncé, mais son affliction me hante encore aujourd'hui.
Chimiothérapie, radiation et mastectomie, métastases aux os et au foie : elle s'est battue pendant 10 ans. Atteinte d'une complication intestinale rare, elle a fini par être admise à l'unité des soins intensifs d'une ville voisine. Je suis allée lui rendre visite un soir afin de prévoir son transfert à domicile pour les soins palliatifs.

Quelques jours plus tard, je suis allée la voir chez elle. Un lit d'hôpital avait été installé dans la cuisine. Les murs étaient d'un doux orangé, de la couleur d'un coucher de soleil. Son lit prenait probablement la place d'une table, où sa famille devait jadis se réunir pour se raconter avec entrain les anecdotes de la journée.

J'ai vu dans ses yeux bruns brillants l'esprit combatif d'une princesse guerrière. "Je ne veux pas mourir », m'a-t-elle chuchoté, dans le doux vrombissement de la machine à oxygène à son chevet. 
J'ai prié pour qu'elle survive, cette jeune et belle mère, cette patiente sur qui le sort s'acharnait. J'ai prié parce que j'étais terrifiée à l'idée de ne pas pouvoir la sauver. Lorsque sa condition s'est détériorée, elle s'est mise à souffrir énormément. J'ai alors cessé de souhaiter qu'elle vive plus longtemps. J'ai plutôt prié pour que la douleur parte, pour que la nausée parte, pour que la peur parte.

Elle n'avait plus l'usage de la parole lorsque son père, les traits pâles et tirés, est arrivé à son chevet. Il s'est penché sur sa fille, puis levant les yeux vers moi, il a dit : «Ça me serre le cœur de savoir qu'elle ne pourra plus jamais me parler. »

Elle a alors entrouvert les yeux et a articulé clairement : « Je t'aime, papa. »

Nous avons versé des larmes en la regardant reprendre sa respiration haletante. On aurait dit qu'elle venait de traverser un pont et qu'elle nous avait laissés de l'autre côté.

Je me souviens que peu après le diagnostic de sa fille, le père avait pris rendezvous avec moi et m'avait demandé : "Comment cette situation a-t-elle pu se produire? " Ses mots calmes avaient résonné en moi comme une accusation, et je me suis sentie encore plus coupable de ne pas pouvoir lui promettre de guérir sa fille.

Je m'en suis voulue pendant 10 ans pour ce délai de diagnostic. Pourtant, lors de notre dernière conversation avant sa mort, ma patiente m'a remerciée. "Merci d'avoir pris soin de moi. "Savait-elle seulement ce que ces mots représentaient pour moi? C'est le plus beau cadeau qu'elle aurait pu me faire, et je ne me sentais pas digne de le recevoir. J'étais impressionnée de voir sa grande combativité faire place à un lâcher-prise avec autant de grâce et de compassion. J'ai eu l'impression qu'elle me faisait ses adieux et qu'elle voulait me montrer qu'elle m'avait pardonné.
L'appel est venu de son mari, au petit matin, après une nuit de vent glacial et d'orages. La sombre route de campagne que j'ai prise pour aller constater son décès à son domicile était jonchée de branches cassées.

Elle était entourée d'équipement médical : un masque à oxygène et des tubes; des suppléments nutritionnels sur la table de nuit; des boîtes de lingettes alcoolisées et de gazes; et une enveloppe scellée d'aiguilles de type papillon. C'est le nom qu'on donne aux minuscules aiguilles utilisées pour injecter de la morphine: des papillons. Ironique, n'est-ce pas? Comme si leur grâce avait quelque chose à voir avec la douleur d'une jeune mère.

Sa sœur a lissé les couvertures autour d'elle comme on borde un tout-petit avant la sieste. Elle pleurait en replaçant doucement une mèche de cheveux sur son front. Nos yeux se sont croisés, et je crois que nous étions toutes les deux soulagées de la savoir libérée des interventions chirurgicales, de la chimiothérapie, des médicaments et des traitements (d'abord pour la guérir, puis pour la soulager et, à la fin, pour gagner du temps). Elle avait l'air sereine lorsque j'ai replié ses mains sur la petite tortue en peluche qui était toujours sur son lit. La maison était calme; ses enfants dormaient paisiblement à l'étage.

Les préposés du salon funéraire ont sorti son corps de la maison sans un bruit. Pour faire passer la civière, ils ont dû débrancher le grand ange de Noël sur le perron. La noirceur semblait définitive jusqu'à ce qu'un des hommes se penche et le rebranche. Il ne restait que 10 jours avant Noël même si le sol, mouillé et boueux, n'avait pas encore gelé. Je me souviens d'avoir pensé qu'il aurait dû neiger, qu'en temps normal, elle aurait été en train de se préparer pour les fêtes avec ses enfants.
Sa mort m'a dévastée. Mon incapacité à la sauver me rappelait que la situation pourrait se reproduire. Je me suis sentie inadéquate, comme un imposteur, incapable de guérir quiconque, encore moins moi-même. Je voyais ma tristesse et ma sensibilité comme une faiblesse, un échec du maintien de mes limites professionnelles avec les patients et les familles sous mes soins, limites pourtant si importantes dans une petite ville.

Ce matin-là, je me suis rendue au travail. Le soleil se levait à l'horizon; la route était encore mouillée et jonchée de branches cassées. Dans le stationnement, je me suis regardée dans le rétroviseur. Le chagrin et la fatigue avaient rendu mes yeux vitreux. En pensant à la journée qui s'en venait, je me suis soudainement sentie impuissante. Impuissante à convoquer à nouveau l'idéalisme de mes jeunes années de pratique, impuissante à trouver du temps pour mon introspection et ma propre guérison. Ma compassion et ma sensibilité faisaient de moi un meilleur médecin, surtout pour les patients que je ne pouvais guérir, mais il était maintenant nécessaire de rediriger une partie de cette compassion vers moi-même.

\section{Sharon McCutcheon MD}

Sussex (Nouveau-Brunswick)

Cet article a été révisé par des pairs.

Il s'agit d'une histoire vraie. Le mari de la patiente a consenti à ce que nous la racontions.

Propriété intellectuelle du contenu : II s'agit d'un article en libre accès distribué conformément aux modalités de la licence Creative Commons Attribution (CC BY-NC-ND 4.0), qui permet l'utilisation, la diffusion et la reproduction dans tout médium à la condition que la publication originale soit adéquatement citée, que l'utilisation se fasse à des fins non commerciales (c.-à-d., recherche ou éducation) et qu'aucune modification ni adaptation n'y soit apportée. Voir : https://creativecommons.org/licenses/by -nc-nd/4.0/deed.fr 